\title{
MODELAGEM CONSTITUTIVA DE ESTRUTURAS REFRATÁRIAS CONSIDERANDO EFEITOS DE DANO *
}

Lucas Breder Teixeira ${ }^{1}$ Samuel Silva Penna ${ }^{27}$

\section{Resumo}

Esse trabalho discute a modelagem termo-mecânica fisicamente não linear de materiais refratários considerando os efeitos de danificação utilizando o Método dos Elementos Finitos. Inicialmente é feita uma descrição geral dos materiais refratários com foco em suas características termo-mecânicas, destacando principalmente suas propriedades elásticas e comportamento de amolecimento, e demonstrando sua considerável dependência da temperatura. Algumas considerações são feitas com relação à evolução dos modelos de simulação numérica aplicados a refratários, iniciando-se com os modelos lineares elásticos até o uso de modelos constitutivos complexos. O modelo de fissuração distribuída utilizado para calcular a perda de rigidez elástica é então apresentado, juntamente com a fundamentação matemática pertinente e sua relação com os parâmetros físicos do material. Para ilustrar a aplicabilidade do modelo alguns exemplos são apresentados, discutindo-se suas principais capacidades com relação ao projeto de estruturas refratárias..

Palavras-chave: Refratários; Fissuração Distribuída; Dano; Elementos Finitos.

\section{CONSTITUTIVE MODELING OF REFRACTORY STRUCTURES CONSIDERING DAMAGE EFFECTS}

\section{Abstract}

This paper discusses the thermomechanical physically non-linear modelling of refractory materials considering the effects of damage using the Finite Element Method. Initially a general description of refractory materials is made focusing in their thermomechanical characteristics, highlighting mainly their elastic properties and softening behaviour, showing the considerable temperature dependency. Some considerations are made about the evolution of the numerical modelling of refractory materials, starting from linear elastic models until the use of complex constitutive models. The smeared cracking model used to calculate the stiffness lost is then presented, within the pertinent mathematical framework and its relation with the physical material parameters. To illustrate the applicability of the model some examples are provided, discussing its main capabilities in terms of design of refractory structures.

Keywords: Refractories; Smeared Cracking; Damage; Finite Elements.

1 Engenheiro Mecânico, Mestrando em Engenharia de Estruturas, Universidade Federal de Minas Gerais, Belo Horizonte, Minas Gerais, Brasil..

2 Engenheiro Civil, Dr., Professor Adjunto, Departamento de Engenharia de Estruturas, Universidade Federal de Minas Gerais, Belo Horizonte, Minas Gerais, Brasil. 


\section{INTRODUÇÃO}

Materiais refratários são projetados para resistirem a altas temperaturas. Em função dessa característica de sua utilização são expostos a tensões mecânicas, térmicas, corrosão, erosão, difusão de gases e abrasão (Banerjee, 2004).

Devido à sua resistência termo-mecânica esse tipo de material é muitas vezes utilizado como revestimento de equipamentos metálicos na indústria siderúrgica, petrolífica, cimenteira, entre outras. Dessa forma, estão sujeitos a carregamentos mecânicos como o peso próprio e o impacto de outros materiais, além de carregamentos térmicos devidos principalmente a três fatores (Gasser et al., 2001):

- Gradiente térmico devido à diferença de temperatura entre a face interna em contato com o produto sendo processado (face quente) e a temperatura externa do equipamento (face fria);

- Diferença entre o coeficiente de expansão térmica dos refratários e da estrutura metálica;

- Ciclagem térmica em função do constante carregamento e descarregamento de produto quente no interior do equipamento (por exemplo, carregamento e vazamento de uma panela de aço utilizada no processo de lingotamento contínuo).

De acordo com a função específica que irá desempenhar, diferentes propriedades mecânicas e térmicas são requeridas aos refratários. Além disso, em diversas situações o material irá interagir com metais líquidos a alta temperatura, outros refratários, materiais isolantes e uma estrutura metálica. Devido a esses fatores os projetos do material e da montagem devem ser feitos em conjunto, levando em conta o tipo específico de aplicação e a operação do equipamento.

A correta determinação das propriedades térmicas dos materiais que compões os equipamentos é essencial para obter boa precisão no cálculo dos gradientes de temperatura que são posteriormente utilizados como base para o cálculo de tensões. Dentre as propriedades térmicas dos refratários destaca-se sua condutividade térmica, difusividade, emissividade e refratariedade, sendo a última definida pelo Annual Book of ASTM Standards (1992) como sendo a "capacidade de manter um desejado grau de identidade química e física a altas temperaturas e em ambientes e condições de uso". Em outras palavras, um material com boa refratariedade mantém suas principais características a altas temperaturas, tornando-o adequado a aplicações onde é exposto a esse tipo de situação.

\subsection{Propriedades Mecânicas dos Materiais Refratários}

As propriedades mecânicas dos materiais refratários são de fundamenal importância para sua modelagem e posterior projeto de estruturas resistentes aos carregamentos termo-mecânicos impostos durante a operação. Dentre essas propriedades, destacam-se:

\subsubsection{Coeficiente de Expansão Térmica}

Para o projeto do revestimento refratário de equipamentos o coeficiente de expansão térmica é uma propriedade particularmente importante. Deve-se levar em conta que a montagem do revestimento é realizada à temperatura ambiente e que em geral a operação é feita a temperaturas mais altas. Por isso, juntas de dilatação 
são muitas vezes utilizadas e devem ser dimensionadas de tal forma que, após a expansão dos materiais, não haja excesso de tensões de compressão devido à interação entre eles e que não haja folga excessiva, o que poderia levar à instabilidade da montagem (Duarte, 2000).

\subsubsection{Comportamento Fisicamente Não Linear e Inelástico}

Schacht (1995) mostra através de curvas obtidas a partir de testes unidimensionais de compressão que, a temperaturas relativamente baixas, os materiais refratários tendem a ter um comportamento frágil, e que ganham ductilidade conforme a temperatura aumenta. A Figura 1-a mostra um exemplo de curva tensão-deformação em compressão para um tijolo sílico-aluminoso, e a Figura 1-b mostra um exemplo de curva tensão-deformação em tração para um tijolo de $\mathrm{MgO}$-espinélio, ficando evidente o amolecimento e a resistência residual após a tensão de pico.

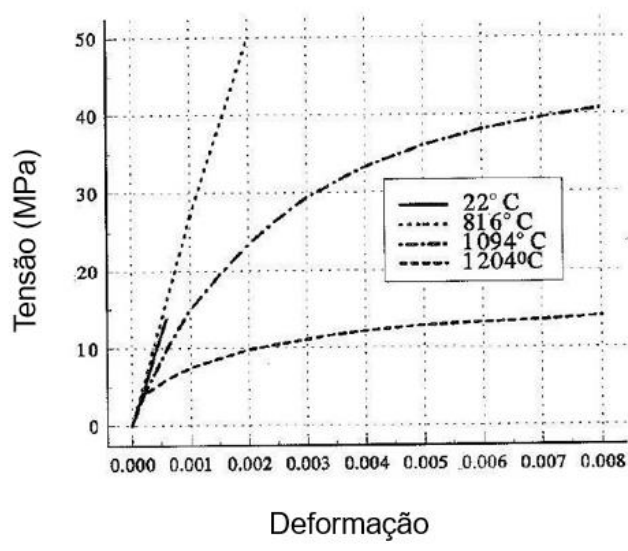

(a)

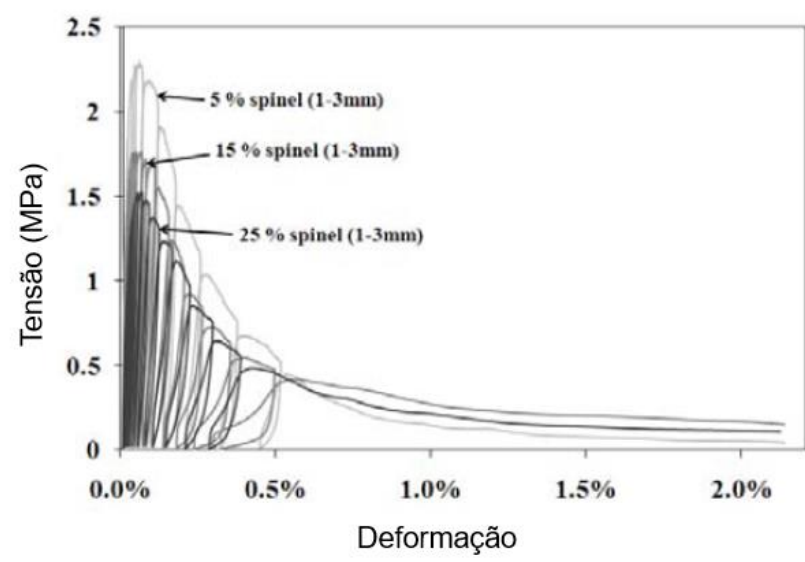

(b)

Figura 1. Exemplos de curvas tensão-deformação em (a) compressão (b) tração.

Segundo Kakroudi (2009), o módulo elástico de materiais refratários depende não somente de seu critério de fabricação, mas também da história de seu carregamento térmico, incluindo a fase de queima. Logo, a rigidez do material tende a variar durante sua vida útil devido ao dano, o que é responsável pelo comportamento inelástico.

\subsubsection{Assimetria Sob Tração e Compressão}

Auer et al. (2005) destacam que as propriedades mecânicas dos materiais refratários variam significativamente de acordo com o estado de tensões a que estão sujeitos, ou seja, são dependentes da pressão hidrostática. Segundo Andreev e Harmuth (2001), a resistência sob um estado triaxial de compressão pode exceder a resistência sob compressão uniaxial em 600\%. Schacht (1995) afirma que a resistência à tração desses materiais não tende a variar significativamente, mas que sob compressão há aumento nas tensões máximas admissíveis. Esses comportamentos são mostrados na Figura 2. 


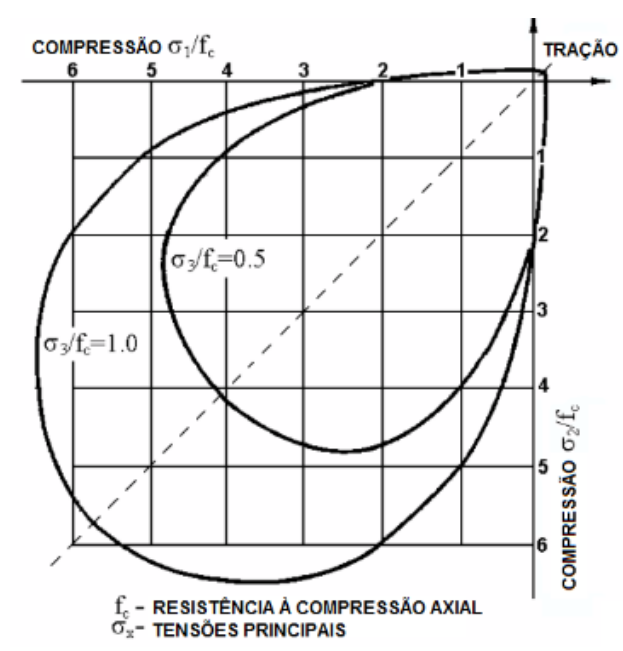

Figura 2. Assimetria sob tração e compressão. Adaptado de Andreev e Harmuth (2001).

\subsubsection{Comportamento em Fratura e Choque Térmico}

Depois de atingida uma tensão crítica sob tração, o material refratário tende a apresentar comportamento de amolecimento, o que resulta em sua capacidade de suportar tensões mesmo após a fratura. Esse tipo de comportamento é ilustrado na Figura 3 para um corpo de prova em um ensaio de flexão em três pontos, retirada de Penna (2011).

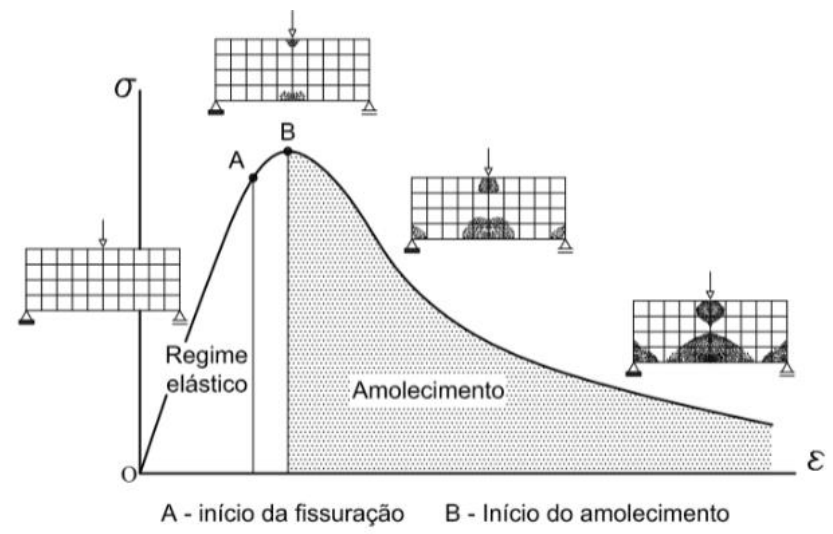

Figura 3. Amolecimento após a tensão de pico (Penna, 2011).

Bradt (2004) mostra que para materiais refratários a região da ponta de uma trinca e principalmente sua esteira são importantes para a compreensão do fenômeno de propagação. Na zona de processo frontal, que contém a ponta da trinca, são observadas microtrincas e múltiplas ramificações. Na região da esteira, o fluxo de silicatos e as pontes de agregados contribuem para uma resistência residual à tração. Esses efeitos são mostrados na Figura e explicam o comportamento não linear sob tração. Isso faz com que os materiais apresentem uma curva ascendente de resistência à propagação (curva $\mathrm{R}$ ), ou seja, quanto maior a trinca, maior a dificuldade de propagação, o que faz com que ela se propague de forma estável antes de ocorrer falha catastrófica. Todas essas considerações explicam a ocorrência do amolecimento após a tensão crítica. 


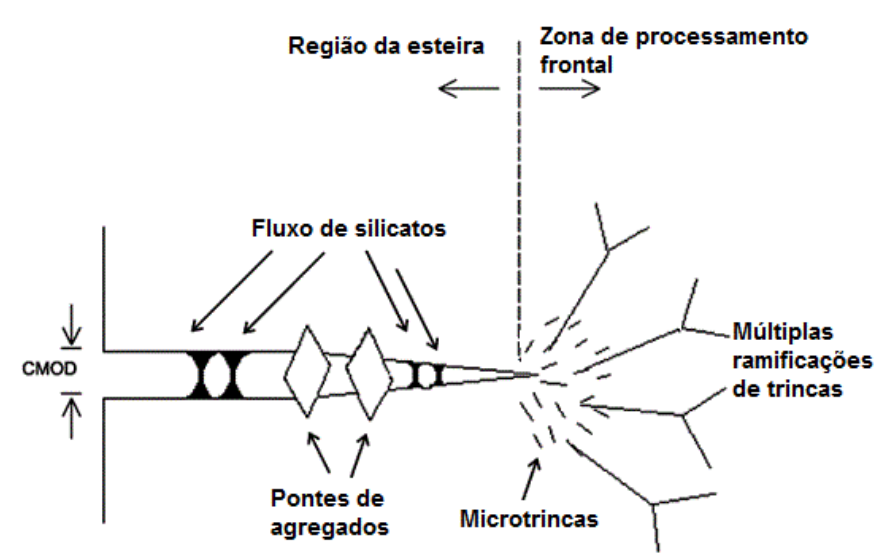

Figura 4. Efeitos observados na esteira e na ponta de uma trinca. Adaptado de Bradt (2004).

\subsubsection{Modelagem de Materiais Refratários}

Na simulação numérica termo-estrutural de materiais refratários a escolha adequada do tipo de modelo constitutivo que será utilizado é de vital importância para o sucesso da análise. Para que haja compatibilidade entre os recursos computacionais disponíveis, o nível de detalhes que devem ser observados e o tempo de projeto, é necessário conhecimento das vantagens e limitações de cada abordagem.

Devido à sua geometria muitas vezes complexa e ao comportamento fisicamente não linear dos materiais refratários a simulação numérica termo-estrutural, usando o método dos elementos finitos, de equipamentos revestidos por esses materiais, enfrenta dificuldades relacionadas ao custo computacional e à convergência dos modelos. Como exemplo, Poirier, Gasser e Boisse (2005) destacam a impossibilidade de modelar uma panela de aço representando todos os seus tijolos, o que os levou a considerá-los como um material monolítico, aumentando consideravelmente as tensões calculadas.

Nguyen et al. (2011) simularam uma montagem plana com um modelo bidimensional, onde consideraram o alívio das tensões nos tijolos promovido pelas juntas de dilatação através da utilização de um material equivalente; os tijolos foram considerados isotrópicos e lineares elásticos, sem levar em conta a variação das propriedades mecânicas com a temperatura. Desde então a simulação numérica de grandes montagens refratárias considerando os efeitos de absorção de tensões pelas juntas de dilatação tem sido objeto de extensivo estudo, tanto em montagens com juntas argamassadas quanto com juntas secas.

Andreev e Harmuth (2001) modelaram o aquecimento e o choque térmico que ocorrem em uma panela de aço utilizarando o critério de Drucker-Prager combinado com um "cut-off" de tração pelo critério de Rankine, e um modelo de fissuração distribuída para modelar o comportamento em tração, considerando a variação das propriedades dos materiais com a temperatura.

Diversos outros trabalhos foram publicados nos ultimos anos relativos à modelagem constitutiva de materiais refratários. No entanto, nota-se que ainda não há consenso sobre qual o melhor modelo a ser utilizado, e modelos simples e funcionais ainda devem ser desenvolvidos.

\section{MATERIAIS E MÉTODOS}

Nesse trabalho são apresentadas características da modelagem não linear de estruturas sujeitas a altas temperaturas utilizando o modelo de fissuração distribuída 
em conjunto com o método dos elementos finitos, com aplicação em materiais refratários. Como plataforma numérica foi utilizado o software INSANE - INteractive Structural ANalysis Environment, criado e desenvolvido no Departamento de Estruturas da Universidade Federal de Minas Gerais (UFMG). Mais detalhes sobre o software podem ser encontrados em www.insane.dees.ufmg.br.

A seguir, uma descrição dos modelos de fissuração distribuída e as particularidades de sua aplicação a materiais refratários.

\subsection{Modelo de Fissuração Distribuída}

A modelagem do processo de fratura pode, de maneira geral, seguir duas abordagens. A primeira diz respeito ao uso de modelos de trinca discreta, onde a falha é introduzida na geometria através da desconexão de nós da malha quando um critério é atingido; algumas desvantagens desse processo são a constante necessidade de remalhamento e seu alto custo computacional, além de poder apresentar incompatibilidade física com a fissuração de materiais parcialmente frágeis que tendem a apresentar microtrincas e perda de rigidez antes do aparecimento de macrotrincas. A segunda abordagem, utilizada nesse trabalho, refere-se à utilização de modelos constitutivos determinados a partir da mecânica do dano e que, mantendo a malha fixa durante toda a análise, promovem a degradação gradual das propriedades elásticas do material.

Segundo Lemaitre e Desmorat (2005), o desenvolvimento do dano no material afeta suas propriedades mecânicas de diversas maneiras, incluindo a diminuição de seu módulo elástico e de sua densidade.

A Figura 5 mostra um corpo onde, em determinado plano de área $\delta S$ e normal $\vec{n}$ em um elemento volumétrico representativo (RVE, na sigla em inglês), foram desenvolvidas fissuras de área total $\delta S_{D}$. Matematicamente, esse plano pode ser representado por uma nova configuração de área efetiva, ou seja, área com capacidade de resistir a esforços, $\delta \tilde{S}$ e normal $\tilde{\vec{n}}$.

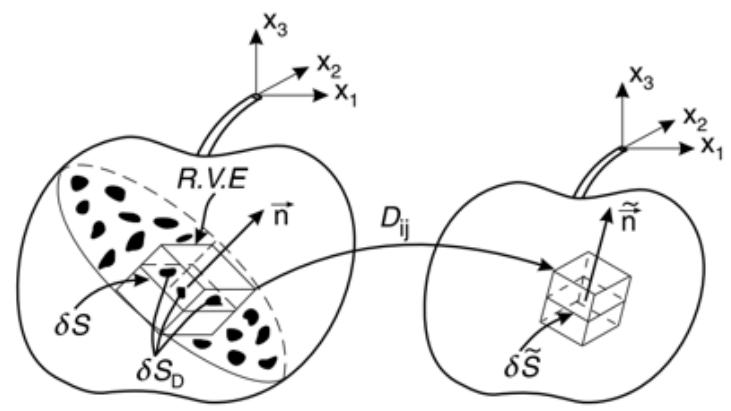

(a)

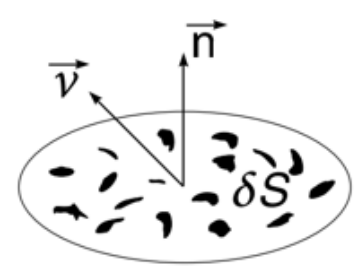

(b)

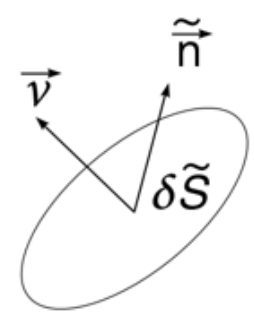

Figura 5. (a) Redução da área efetiva de um material sujeito a dano e sua representação matemática. (b) Detalhe. Adaptado de Lemaitre e Desmorat (2005). 
É possível definir então uma variável de dano que depende da normal $\vec{n}$ com a forma:

$$
D_{(\vec{n})}=\delta S_{D} / \delta S
$$

Dependendo do caráter da fissuração a variável de dano pode ser considerada isotrópica, ou seja, com o dano se manifestando igualmente em todas as direções, ou ortotrópica, onde cada uma das três direções espaciais tem comportamento distinto. Uma característica dos modelos de fissuração é que inicialmente adota-se uma hipótese de que o meio material é inicialmente linear, elástico, isotrópico e homogêneo, e durante o carregamento e consequente evolução da variável de dano o meio se torna não-linear, inelástico, ortotrópico, mas ainda homogêneo (Penna, 2011).

Para considerar a danificação do meio, os modelos de fissuração distribuída atuam no sentido da redução da rigidez do material. Dessa maneira, após aplicado o carregamento, a rigidez residual é dada por:

$$
\tilde{E}=(1-D) \times E
$$

onde $D$ é a variável que acompanha a evolução do dano.

Em seu estado mais atual os modelos de fissuração distribuída partem de uma relação de flexibilidade entre deformação e tensão definida no sistema local do plano de aparecimento da trinca. Através da aplicação de um esquema de simetrização da matriz de flexibilidade (Penna, 2011), é definido um tensor constitutivo secante local dado no caso 2D por:

$$
{ }_{l}^{s} D=\frac{1}{1-\frac{E_{n} E_{S}}{E_{0}^{2}} v^{2}}\left[\begin{array}{ccc}
\frac{E_{n}}{v E_{n} E_{S}} & \frac{v E_{n} E_{S}}{E_{0}} & 0 \\
E_{0} & E_{S} & 0 \\
0 & 0 & \left(1-\frac{E_{n} E_{S}}{E_{0}^{2}} v^{2}\right) G_{n s}
\end{array}\right]
$$

onde $E$ é o módulo elástico, $v$ o coeficiente de Poisson, $n$ e $s$ são as direções principais de ortotropia e $G_{n s}$ o módulo de cisalhamento, dado por:

$$
G_{n s}=\frac{E_{0} E_{n} E_{S}}{E_{0} E_{n}+E_{0} E_{s}+2 v E_{n} E_{s}}
$$

Para considerar os efeitos de rugosidade da fissura e engrenamento dos agregados o módulo de elasticidade transversal deve ser limitado por:

$$
G_{n s} \geq \beta G_{0}
$$

com

$$
G_{0}=\frac{E_{0}}{2(1+v)}
$$

Tendo definido o tensor constitutivo secante local, um importante passo para 0 equacionamento do modelo de fissuração distribuída é sua transformação para o 
sistema de coordenadas global. Essa etapa é necessário pois as equações do método dos elementos finitos são resolvidas de acordo com um sistema global de referência, e não no sistema de deformações principais relativos ao plano da trinca. Após a definição matemática do modelo constitutivo torna-se necessária a adaptação das curvas tensão-deformação do material a leis constitutivas previamente estabelecidas, etapa chamada de calibração. No caso dos materiais refratários a calibração deve ser realizada para cada temperatura na qual haja dados disponíveis. Na seção seguinte será mostrada a calibração das leis constitutivas de um material refratário utilizado como exemplo para a realização desse trabalho.

\subsection{Leis Constitutivas para o Material Refratário}

Conforme explicado nas seções anteriores, materiais refratários tem propriedades mecânicas altamente dependentes da temperatura, além de possuírem comportamento diferente sob tração e sob compressão. Dessa maneira, a Figura 6 mostra as curvas tensão-deformação utilizadas para os exemplos de modelagem realizados nesse trabalho, calibradas utilizando-se o modelo de Carreira para compressão e Ingraffea para tração (Penna, 2011). A Tabela 1 mostra os parâmetros utilizados para o material simulado.

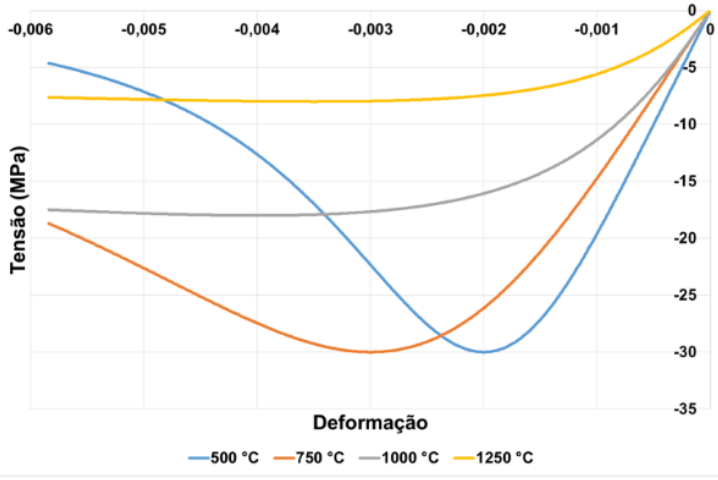

(a)

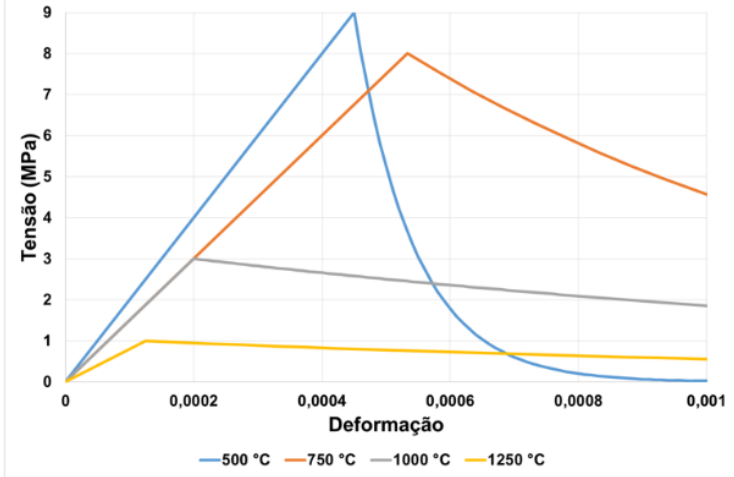

(b)

Figura 6. Curvas tensão-deformação utilizadas para a modelagem (a) Compressão (b) Tração.

Tabela 1. Parâmetros dos materiais - modelo de Carreira e Ingraffea

\begin{tabular}{ccccc}
\hline & \multicolumn{4}{c}{ Temperatura $\left({ }^{\circ} \mathbf{C}\right)$} \\
\hline $\boldsymbol{v}$ & $\mathbf{5 0 0}$ & $\mathbf{7 5 0}$ & $\mathbf{1 0 0 0}$ & $\mathbf{1 2 5 0}$ \\
\hline $\boldsymbol{f}_{\boldsymbol{c}}(\mathrm{MPa})$ & 0,2 & 0,2 & 0,2 & 0,2 \\
\hline $\boldsymbol{f}_{\boldsymbol{t}}(\mathrm{MPa})$ & 9 & 30 & 10 & 8 \\
\hline $\boldsymbol{\varepsilon}_{\boldsymbol{c}}$ & 0,002 & 0,002 & 0,004 & 0,0035 \\
\hline $\boldsymbol{E}_{\boldsymbol{0}}(\mathrm{MPa})$ & 20000 & 15000 & 15000 & 8000 \\
\hline $\boldsymbol{g}_{\boldsymbol{f}}(\mathrm{N} / \mathrm{m})$ & $5 \mathrm{e}-5$ & $4 \mathrm{e}-4$ & $1,5 \mathrm{e}-4$ & $3 \mathrm{e}-5$ \\
\hline $\boldsymbol{h}(\mathrm{m})$ & 0,06 & 0,06 & 0,03 & 0,02 \\
\hline $\boldsymbol{\beta}$ & 0,05 & 0,05 & 0,05 & 0,05 \\
\hline
\end{tabular}

$\boldsymbol{f}_{\boldsymbol{c}}=$ Resistência à compressão; $\boldsymbol{f}_{\boldsymbol{t}}=$ Resistência à tração; $\boldsymbol{\varepsilon}_{\boldsymbol{c}}=$ Deformação no pico de compressão; $\boldsymbol{g}_{\boldsymbol{f}}=$ Energia de fratura; $\boldsymbol{h}=$ Comprimento característico; $\boldsymbol{\beta}=$ Fator de retenção ao cisalhamento

É importante observar que, tomando-se a curva a $500^{\circ} \mathrm{C}$ como exemplo, a resistência mecânica à compressão é mais que 3 vezes maior para o corpo sujeito à compressão que à tração, e que o material tende a ser "menos frágil" conforme a temperatura aumenta, o que leva as trincas a se propagarem de maneira menos 
catastrófica e permitirem que a estrutura tenha uma resistência residual menos após ser atingido o pico de carga.

\subsection{Exemplos Simulados}

Para demonstrar as aplicações do modelo são apresentados exemplos onde a danificação do material é um parâmetro importante e a maneira como a estrutura se comporta depende da distribuição de temperaturas.

\subsubsection{Viga em Flexão Sujeita a Diferentes Distribuições de Temperaturas}

O modelo testado corresponde a uma viga de dimensões 1,92m x 0,48 $\mathrm{m} \times 36 \mathrm{~mm}$, composta pelo material descrito na Seção 2.2 e sujeita a um carregamento de $1 \mathrm{MN}$ no centro de seu vão. Conforme mostrado na Figura 7, foram impostas três condições distintas de carregamento, todas com temperaturas variando entre $1250^{\circ} \mathrm{C}$ e $500^{\circ} \mathrm{C}$, sendo:

- Condição 1: Região da viga sujeita a tração tendo a maior temperatura (Figura 7-b);

- Condição 2: Região da viga sujeita a tração tendo a menor temperatura (Figura 7-c);

- Condição 3: Temperatura distribuída lateralmente na viga.

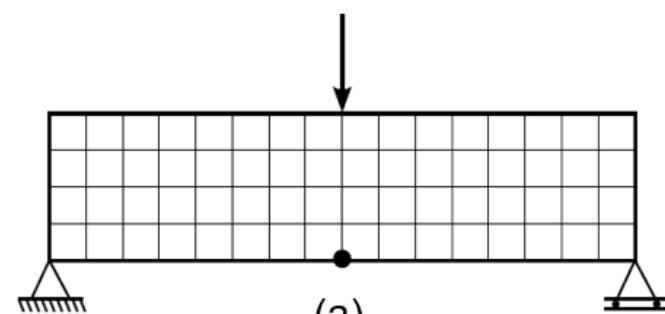

(a)

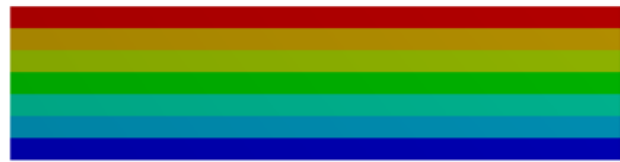

(c)

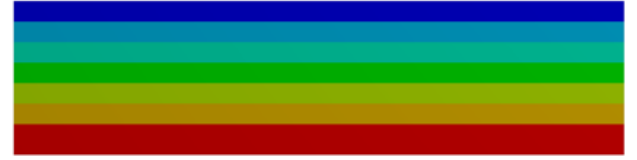

(b)

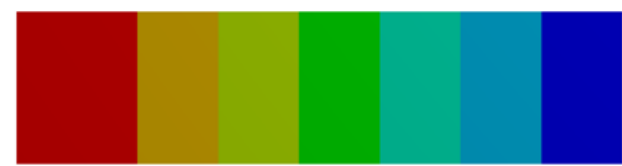

(d)

Figura 7. Modelo de viga em flexão (a) Malha e nó de avaliação da trajetória de equilíbrio. (b) Gradiente de temperaturas de baixo para cima. (c) Gradiente de temperaturas de cima para baixo. (d) Temperatura distribuída lateralmente.

Para obter a solução foi utilizado um método de controle do deslocamento horizontal do nó destacado na Figura 7-a. Foi utilizado um fator de carga de $5 \times 10^{-6} \mathrm{~m} \mathrm{e}$ tolerância de $1 \times 10^{-4}$. Para a realização dos cálculos foi utilizado o software INSANE.

\section{RESULTADOS E DISCUSSÃO}

\subsection{Viga em Flexão}


A Figura 8 mostra as curvas Fator de Carga x Deslocamento Vertical do Nó de Referência obtidas para os casos com distribuição de temperaturas mostradas na Figura 7, além de curvas relativas a vigas com temperaturas constantes de $500^{\circ} \mathrm{C}$ e $1250^{\circ} \mathrm{C}$, que podem ser utilizadas como referência na análise.

É possível observar que, quando a viga está a $500^{\circ} \mathrm{C}$, a carga máxima suportada é de aproximadamente $3 \%$ da carga de referência aplicada, ou seja, $30 \mathrm{kN}$, porém o deslocamento máximo chega apenas $-0,86 \mathrm{~mm}$ antes que haja perda de estabilidade no modelo, o que indica falha catastrófica da estrutura.

A $1250^{\circ} \mathrm{C}$ a viga possui menor resistência mecânica, no entanto sua fragilidade é bastante reduzida, fazendo com que suas trincas se propaguem de maneira mais estável e permita que a estrutura tenha uma resistência residual após o pico de carga, chega a deformações de $-3,0 \mathrm{~mm}$.

O comportamento nas demais condições de distribuição de temperaturas é intermediário aos discutidos anteriormente. Conforme esperado, quando a região de tração está sujeita à temperatura mais baixa (Figura 7-c), portanto seu material tendo maior resistência mecânica e menor energia de fratura, a tendência é que a resposta da estrutura esteja próxima à da viga a $500^{\circ} \mathrm{C}$. Quando a distribuição de temperaturas se inverte (Figura 8-b) a viga adquire ductilidade e consegue, mesmo apresentando a característica de suportar maiores deformações, apresentar uma resistência mecânica $63 \%$ superior à viga com temperatura constante de $1250^{\circ} \mathrm{C}$. Submetendo-se a viga à distribuição lateral de temperaturas consegue-se igualmente obter uma boa resistência mecânica na estrutura, no entanto a carga máxima admissível decresce com maior intensidade.

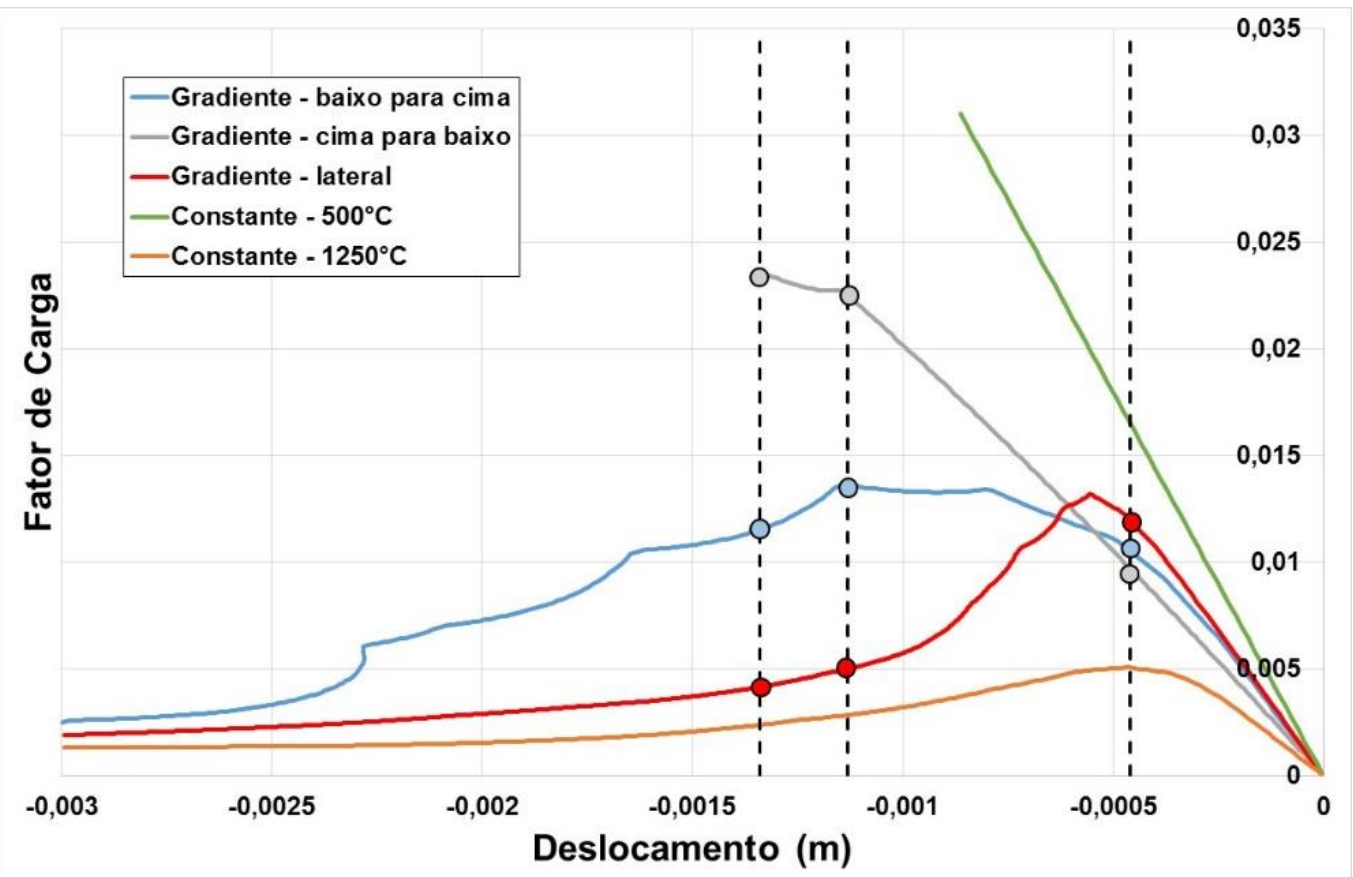

Figura 8. Trajetórias de equilíbrio para a viga em flexão com diferentes distribuições de temperaturas

Um importante parâmetro que pode ser utilizado como critério de projeto de estruturas refratárias é seu estado de danificação, ou seja, quanto de sua rigidez foi perdida devido ao carregamento. Para o caso de estruturas refratárias, a perda de rigidez do material e o aparecimento de trincas distribuídas pela estrutura contribuem, por exemplo, para o aumento da infiltração de aço líquido em um 
processo siderúrgico, resultando em aumento da corrosão e da erosão, somadas à perda da capacidade de resistir a carregamentos mecânicos.

A Figura 9 mostra a evolução do estado de danificação da viga com o carregamento, em diferentes distribuições de temperaturas. Os pontos onde foram feitas as avaliações do dano estão destacados na Figura 8. Nota-se que no caso onde a distribuição de temperaturas é feita conforme a Figura 7-b o centro da viga já apresenta estágio avançado de degradação quando o deslocamento do ponto central é de $-1,13 \mathrm{~mm}$, podendo este ser considerado um critério para afastamento da estrutura. Quando as temperaturas estão distribuídas conforme a Figura 7-c a danificação é consideravelmente menor, no entanto torna-se catastrófica ao atingir o valor máximo, podendo representar um risco à segurança da operação. Quando a distribuição de temperaturas é lateral o dano concentra-se mais à esquerda do centro da viga, tornando este o principal ponto de atenção durante o projeto.

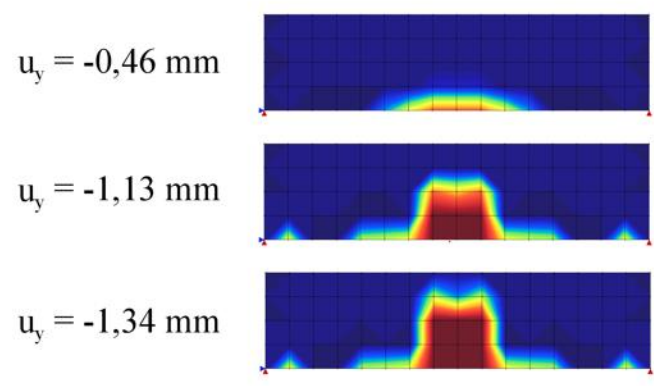

(a)

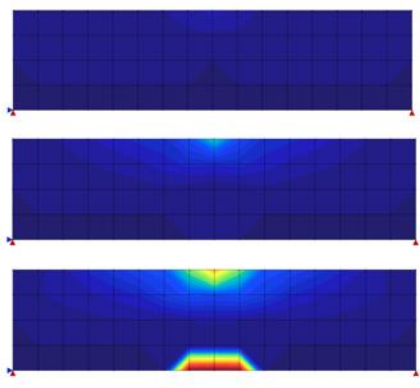

(b)

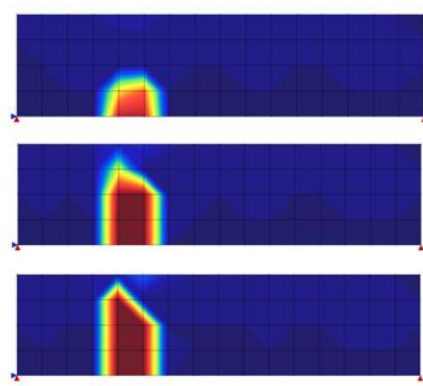

(c)

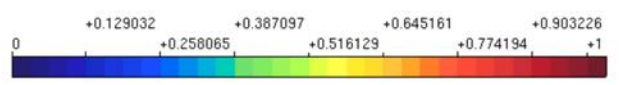

Figura 9. Evolução do estado de danificação da viga com o carregamento, em diferentes distribuições de temperaturas. (a) Gradiente de temperaturas de baixo para cima. (b) Gradiente de temperaturas de cima para baixo. (c) Temperatura distribuída lateralmente.

\section{CONCLUSÃO}

Esse trabalho discutiu a modelagem termo-mecânica de materiais refratários e o projeto de estruturas refratárias levando em consideração efeitos de danificação. Percebe-se que esse tipo de material apresenta comportamento complexo e altamente dependente da temperatura, fazendo com que esse parâmetro tenha influência direta na vida da estrutura durante a operação. É possível concluir que, de acordo com a rotina de operação da estrutura, sendo ela sujeita prioritariamente a cargas de força ou de deslocamento, deve-se fazer a escolha pela utilização de materiais mais frágeis e com maior resistência mecânica ou "menos frágil" e menor resistência mecânica. Por fim, o estado de danificação pode ser levado em conta como critério de projeto e definição da vida de estruturas refratárias, permitindo previsões mais assertivas e que considerem que a estrutura, mesmo tendo atingido ao longo de sua operação altos valores de carga, pode ainda possuir resistência residual.

\section{REFERÊNCIAS}

1 Banerjee, S. - Properties of refractories. 2004; in - C. A. Schacht, ed., Refractories Handbook, Marcel Dekker, Inc., New York, chapter 1, 1-10. 
2 Gasser, A., Boisse, P., Dutheillet, Y. and Poirier, J., Experimental and numerical analyses of thermomechanical refractory lining behaviour. Proceedings of the Institution of Mechanical Engineers, Part L - Journal of Materials: Design and Applications. 2004, 41-54.

3 Annual Book of ASTM Standards, Vol. 15.01: Refractories, Activated Carbon, Advanced Ceramics. 1992. ASTM International.

4 Duarte, A. K. - Testes e propriedades referentes a refratários; 2000; in Magnesita Refratários S.A., ed., Refratários para siderurgia - Aciaria Elétrica, Apostila produzida pelos autores, Contagem.

5 Schacht, C. A. - Refractory linings: Thermomechanical design and applications. 1995; Marcel Dekker, Inc, New York.

6 Kakroudi, M. G., Yeugo-Fogaing, E., Huger, M., Gault, C. and Chotard, T. - Influence of the thermal history on the mechanical properties of two alumina based castables, Journal of the European Ceramic Society 29(15). 2009; 3197 -3204.

7 Auer, T., Gruber, D., Harmuth, H. and Triessnig, A. - Numerical investigations of mechanical behaviour of refractories, in J. D. Smith, ed., Proceedings of the Unified International Technical Conference on Refractories (UNITECR). 2005; Wiley, Orlando, USA.

8 Andreev, K. and Harmuth, H. - Lining design for vessels of the steel industry-application of Finite element calculations. 2001; in Proceedings of 10th International Metallurgical and Materials Conference, República Checa.

9 Penna, S. S. - Formulaçao multipotencial para modelos de degrada çao elástica: Unificação teórica, proposta de novo modelo, implementação computacional e modelagem de estruturas de concreto, Tese de Doutorado, Universidade Federal de Minas Gerais, Belo Horizonte, Brasil. 2001

10 Bradt, R. C. - Fracture of refractories, in C. A. Schacht, ed., Refractories Handbook, Marcel Dekker, Inc., New York, chapter 2. 2004; pp. 11-38.

11 Poirier, J., Gasser, A. and Boisse, P. - Thermo-mechanical modelling of steel ladle refractory structures, Interceram 54. 2005; 182-189.

12 Nguyen, T. M. H., Blond, E., Gasser, A. and Prietl, T. - Mechanical behaviour of masonry with dry joints. 2011; in Proceedings of the Unified International Technical Conference on Refractories (UNITECR), Kyoto, Japan.

13 Lemaitre, J. and Desmorat, R. - Engineering Damage Mechanics, Springer, Cachan, França. 2005. 